\title{
The Red Edge of the Cepheid Instability Strip
}

\author{
Yan Li \\ Yunnan Observatory, Chinese Academy of Sciences \\ P.O. Box 110, Kunming 650011, P.R. China
}

\begin{abstract}
Recent results of investigations of the nonequilibrium effects between gas and radiation on the red edge of the Cepheid instability strip are presented.
\end{abstract}

\section{Introduction}

Many Cepheid investigations focus on the red edge of the instability strip. Deupree (1977) and Stellingwerf (1982) confirm that if time-dependent convection is considered, stars that are sufficiently cool return to a stable state. However, many uncertainties lie in the treatment of convection and we are not completely sure that this treatment correctly describes the interaction of convection with pulsation. Therefore other explanations of the red edge are of great significance. Presented here are our recent results of investigations of the non-equilibrium effects between gas and radiation on the red edge of the Cepheid instability strip.

\section{Results}

Two stellar models with masses of $9 M_{\odot}$ and $5 M_{\odot}$ and Population I chemical composition $(Y, Z)=(0.24,0.021)$ were evolved to the instability strip. The linear stability analysis was carried out for the first three radial modes using Li's (1992a) method, a treatment based on the frozen convection approximation $\left(\nabla \cdot F_{C}^{\prime}=0\right)$ which precisely describes the process where the gas absorbs or emits radiation.

Numerical results show that all of the modes that have been considered are pulsationally unstable in a certain range of temperature with definite blue and red edges. Fig. 1 gives the instability strip of the fundamental mode. The blue edge obtained is in good agreement with that obtained by Iben \& Tuggle (1975). The most striking characteristic is the emergence of the red edge. This contradicts the common opinion that unless a proper treatment of convection is included the red edge is not found. The first and second overtones also have complete instability strips, and the blue edge occurs at progressively higher temperatures for successively higher order modes while the red edge is at almost the same place for all of the three modes.

The work function of the first overtone is displayed in Fig. 2 for the model of $9 M_{\odot}$ at the center of the instability strip during the second crossing. The pulsation is excited by the frozen temperature mechanism ( $\mathrm{Li} \mathrm{1992b)} \mathrm{operating} \mathrm{in} \mathrm{the} \mathrm{hydro-}$ gen and helium ionization zones. In the above regions, the ionization restrains the 
variation of gas temperature. 'Therefore the gas absorbs or emits radiation upon compression or expansion as the gas temperature is lower or higher than the radiation temperature respectively. As the absorbed energy is more than the emitted one, the difference is transformed into pulsation energy.

We suggest that the red edge of the instability strip results from the emergence of convection in the stellar envelope. With the cooling of the star, the successively increasing convection brings more and more energy and gradually replaces the radiative transfer as the primary means of energy transportation. The sharp decline of the radiative luminosity reduces the supply of energy to be absorbed by the gas and then transformed into pulsation energy. As a result, the variation of radiative temperature is restricted, and the efficiency of the frozen temperature mechanism goes down. This effect can be seen in Fig. 2, which shows as well the work function of the same mode for the same model but at the red edge of the instability strip. The contribution of the second helium ionization driving is depressed at the center of the ionization zone, where convection is most developed. In the hydrogen and first helium ionization zones, convection is so strong that the driving effects only appear at the edges of the ionization zones. When the star is even cooler, there are no driving effects left in the ionization zones and the star returns to the static state.
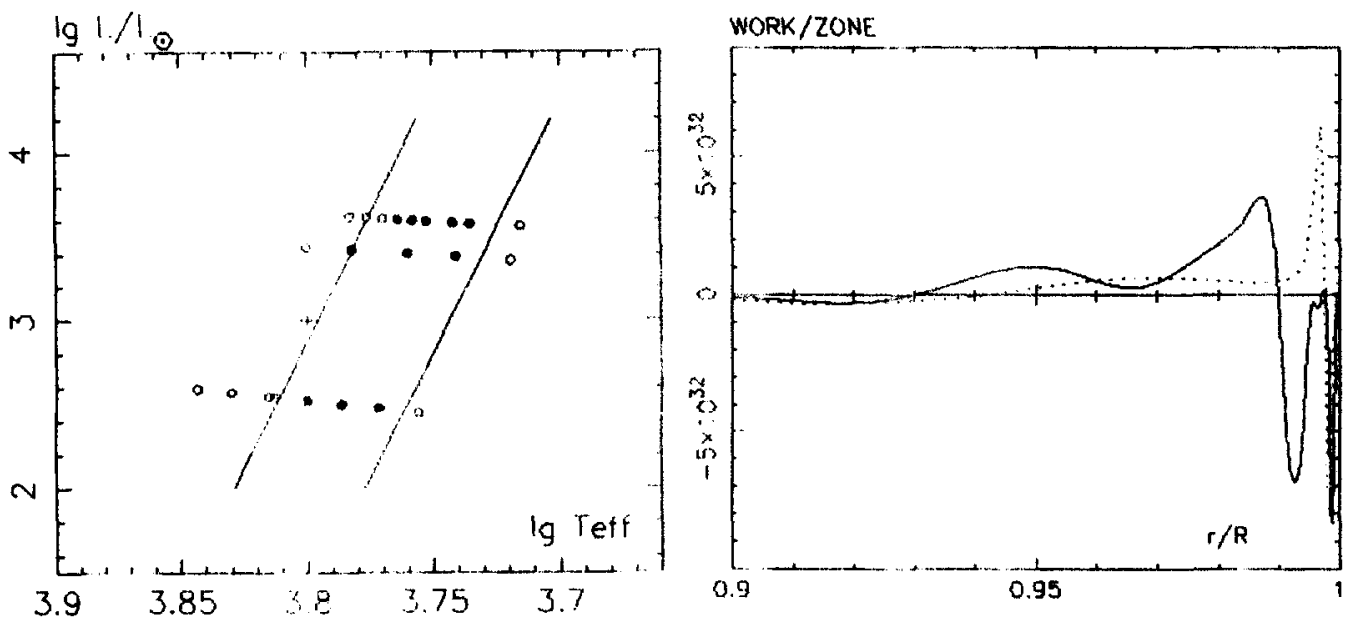

Figure 1. (Left) Instability strip of the fundamental mode: open circles - stable; filled circles - unstable; plus sign - Iben \& Tuggle (1975). Figure 2. (Right) Work function for the first overtone: dotted curve - at the center of the instability strip; solid curve - at the red edge of the instability strip.

\section{Conclusions}

Taking into account the non-equilibrium effects between gas and radiation we obtain the red edge of the instability strip without considering the interaction between convection and pulsation. Therefore, we do not need to use very crude treatment of 
convection-pulsation interaction to account for the red edge of the Cepheid instability strip.

I would like to thank Dr. J. Nemec and Dr. D.L. Welch for their cordial invitation to this colloquium, and for the IAU travel grant. This research has been supported by the National Natural Sciences Foundation grant IEC(92)352.

\section{References:}

Deupree, R.G. 1977, ApJ 211, 509.

Iben, I., Jr. \& Tuggle, R.S. 1975, ApJ 197, 39.

Li, Y., 1992a, A\&A 257, 133.

Li, Y., 1992b, A\&A 257, 145.

Stellingwerf, R.F. 1982, ApJ 262, 339. 